\title{
New Insight into the Hierarchical Microsphere Evolution of Organic Three- Dimensional Layer Double Hydroxide: the Key Role of the Surfactant Template
}

Ping Zhang, ${ }^{* \dagger}$ Xianzhe Zeng, ${ }^{\dagger}$ Peng Li,,$\stackrel{\dagger}{\dagger}$ Tao He,${ }^{\dagger}$ Yun Huang ${ }^{* \dagger}$

${ }^{\dagger}$ Key Laboratory of Poyang Lake Environment and Resource Utilization, Ministry of Education, School of Environmental and Chemical Engineering, Nanchang University,

Nanchang 330031, China

$¥$ Australian Institute for Bioengineering and Nanotechnology, The University of

Queensland, Brisbane, QLD 4072, Australia

*Corresponding authors:

Ping Zhang, E-mail: zhangping@ncu.edu.cn

Yun Huang, E-mail: huangyun@ncu.edu.cn 
Section 1. In detail, $50 \mathrm{~mL}$ of mixed aqueous solution containing $0.16 \mathrm{~mol} \cdot \mathrm{L}^{-1}$ urea, $0.016 \mathrm{~mol} \cdot \mathrm{L}^{-1} \mathrm{Mg}^{2+}$ and $0.008 \mathrm{~mol} \cdot \mathrm{L}^{-1} \mathrm{Al}^{3+}$ was immediately added into various initial concentrations of SDS solution $(30 \mathrm{~mL})$. The phase regions were determined by conductivity measurements. After mixture, the specific SDS concentrations were calculated as follows:

$$
\mathrm{C}_{S D S}=\frac{\mathrm{C}_{s e t} * 30}{80}=0.375 \mathrm{C}_{\text {set }}
$$

Where $C_{S D S}\left(\mathrm{mg} \cdot \mathrm{L}^{-1}\right)$ is the actual SDS concentration, $C_{\text {set }}\left(\mathrm{mg} \cdot \mathrm{L}^{-1}\right)$ is the initial SDS concentration.

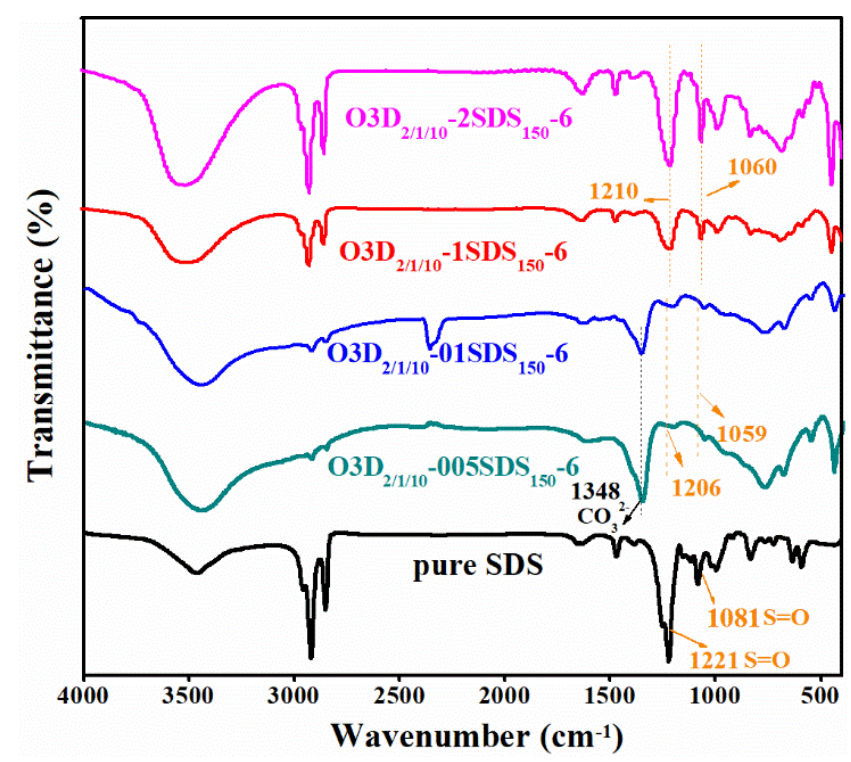

Figure S1. FT-IR spectra of O3D-LDH samples with different SDS concentrations.

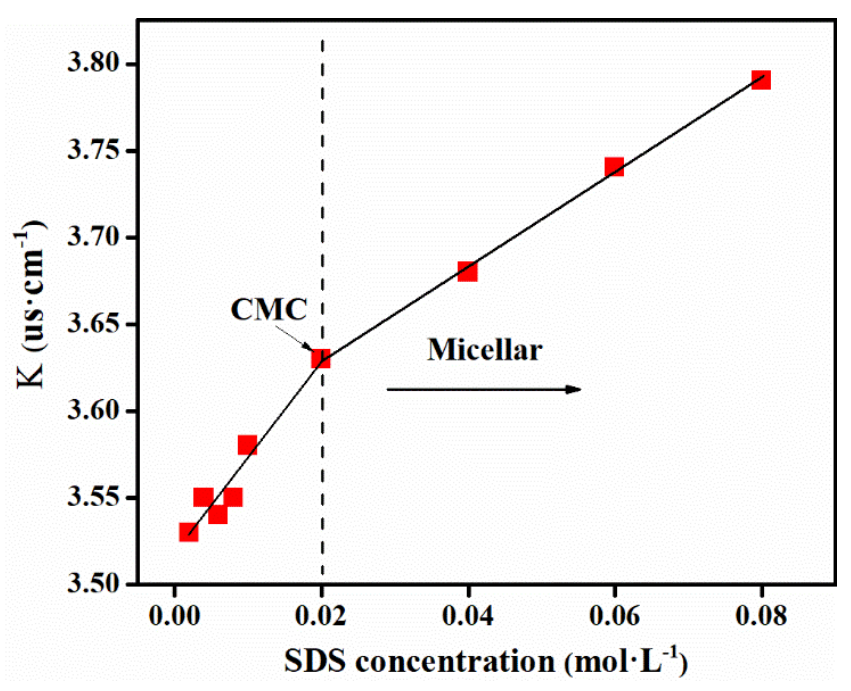


Figure S2. A phase diagram of $[\mathrm{Mg}, \mathrm{Al}$, urea $] / \mathrm{SDS}$ system with varied SDS concentrations.

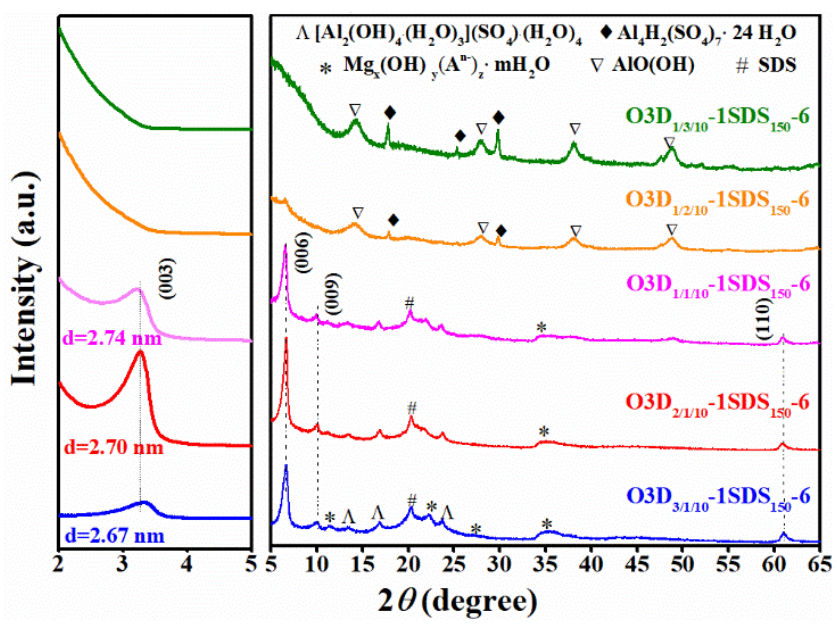

Figure S3. XRD patterns of O3D-LDH samples with different $\mathrm{Mg} / \mathrm{Al}$ ratios.

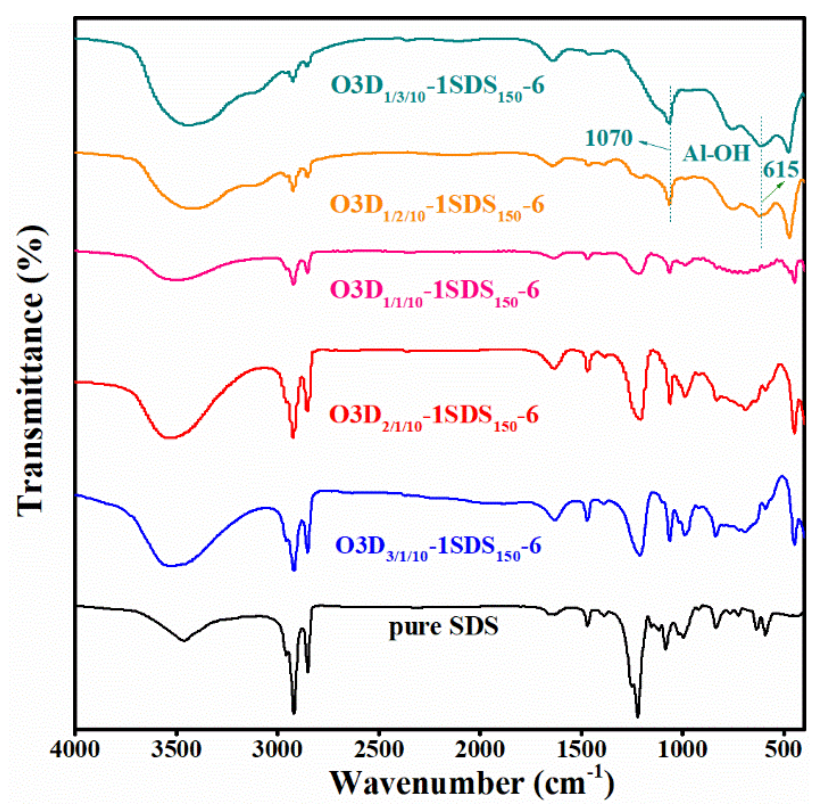

Figure S4. FT-IR spetra of O3D-LDH samples with different $\mathrm{Mg} / \mathrm{Al}$ ratios. 


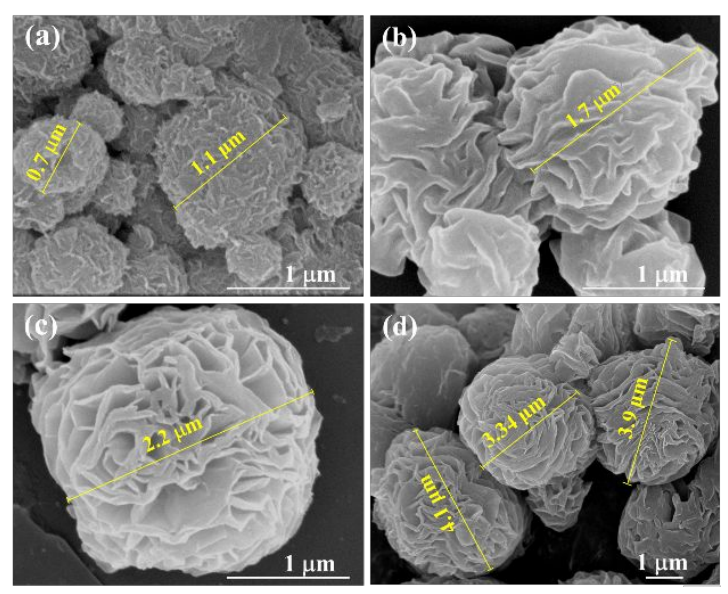

Figure S5. SEM images of O3D-LDH with different hydrothermal temperatures: (a) $\mathrm{O}_{2} \mathrm{D}_{2 / 1 / 10^{-}}-1 \mathrm{SDS}_{100^{-}} 6$, (b) $\mathrm{O}_{2 / 1 / 10^{-}}-\mathrm{SDS}_{125^{-}}$, (c) $\mathrm{O}_{2} \mathrm{D}_{2 / 1 / 10^{-}}-1 \mathrm{SDS}_{150^{-}} 6$, (d) $\mathrm{O}_{2} \mathrm{D}_{2 / 1 / 10^{-}}$ $1 \mathrm{SDS}_{180^{-}} 6$.

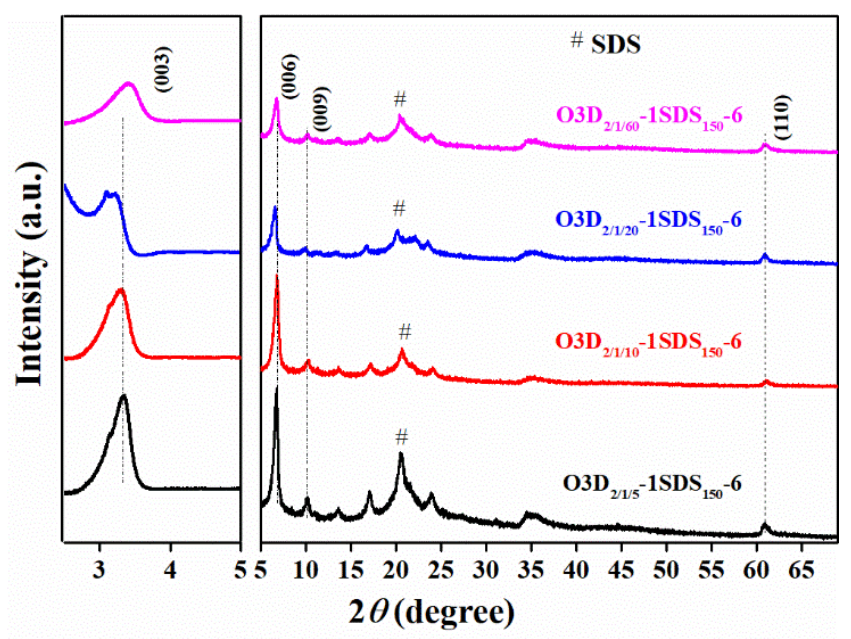

Figure S6. XRD patterns of O3D-LDH samples with different urea concentrations.
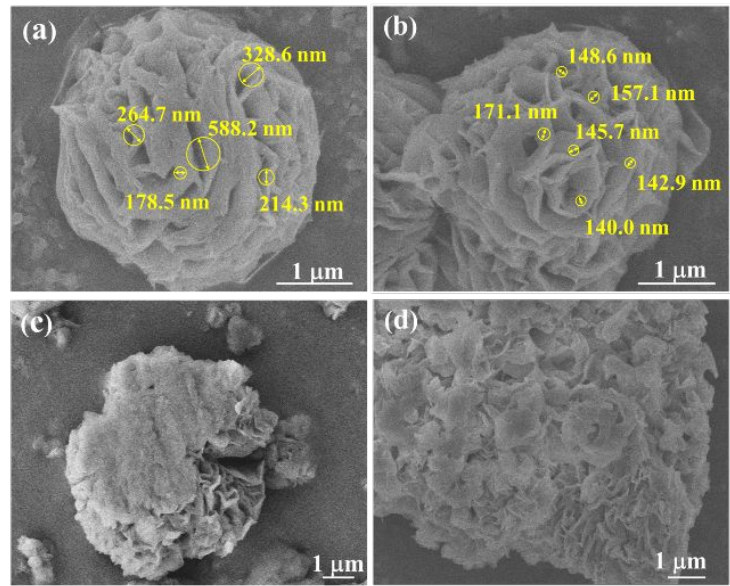
Figure S7. SEM images of O3D-LDH at different urea concentrations: (a) $\mathrm{O}_{2} \mathrm{D}_{2 / 1 / 5^{-}}$ $1 \mathrm{SDS}_{150^{-}} 6$, (b) $\mathrm{O}_{2} \mathrm{D}_{2 / 1 / 10^{-}} 1 \mathrm{SDS}_{150^{-}} 6$, (c) $\mathrm{O}_{2 / 1 / 20^{-}}-\mathrm{SDS}_{150^{-}} 6$, (d) $\mathrm{O}_{2} \mathrm{D}_{2 / 1 / 60^{-}}-1 \mathrm{SDS}_{150^{-}}-6$.

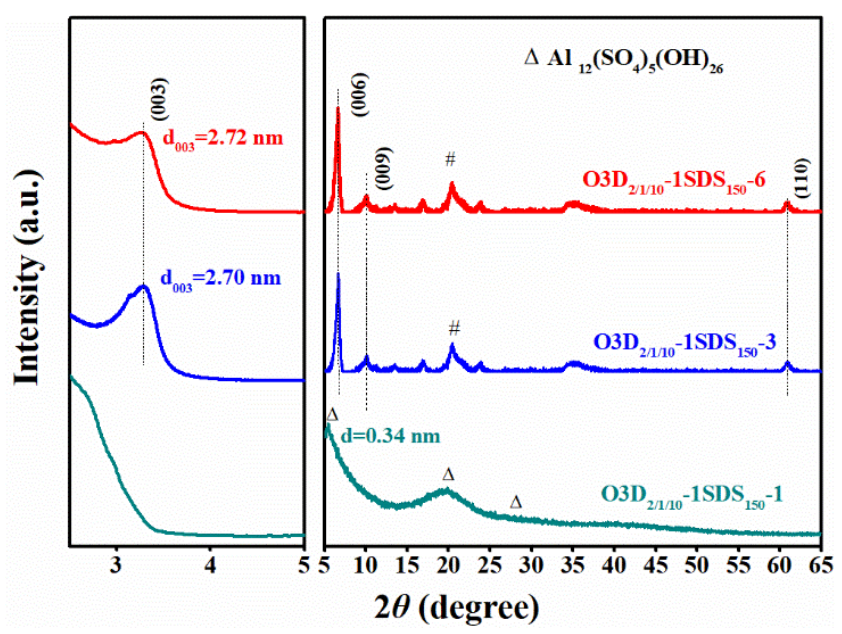

Figure S8. XRD patterns of O3D-LDH at different hydrothermal times.

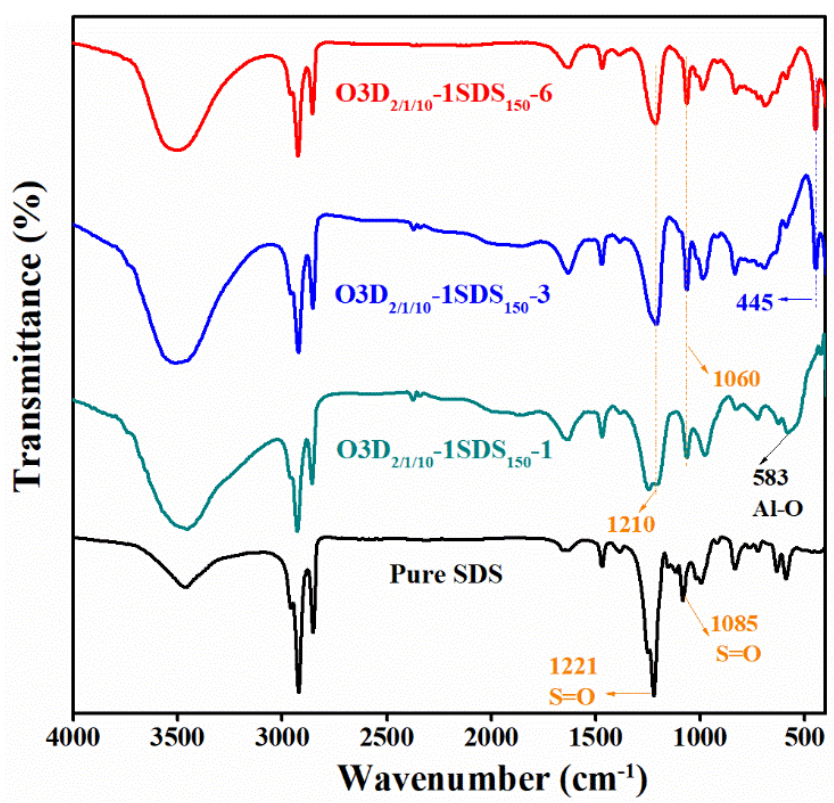

Figure S9. FT-IR spectra of O3D-LDH at different hydrothermal times ranged from 4000 to $400 \mathrm{~cm}^{-1}$. 